\title{
A seismic vertical vibrator driven by linear synchronous motors
}

\author{
Rik Noorlandt ${ }^{1}$, Guy Drijkoningen ${ }^{2}$, Johan Dams ${ }^{3}$, and Rob Jenneskens ${ }^{3}$
}

\begin{abstract}
A linear synchronous motor (LSM) is an electric motor that can produce large controllable forces and is therefore suitable as a driving engine for a seismic vibrator. This motor consists of two independent elements, a magnet track and a coil track, allowing practically unlimited motor displacements. This makes the LSM very suitable for expanding the source frequency band to the lower frequencies in which larger strokes are needed. In contrast to hydraulic engines, the LSM performs equally well over the whole frequency range, making possible a smaller amount of signal distortion, especially at the low frequencies. To find the feasibility of an LSM-driven vibrator, we successfully designed and built a multi-LSM prototype vibrator of some $1200 \mathrm{~kg}$. We addressed the synchronization between the individual motor tracks and the different motors. To lower the energy consumption, a spring mechanism was implemented that delivered the force needed to lift the vibrator mass to its neutral position. The resonance belonging to this spring mechanism was successfully suppressed with the help of a position feedback control that also suppressed the temperature effects. The seismic data acquired in the field tests proved that the prototype LSM vibrator acted very well as a seismic source. It has no trouble generating pseudorandom sweeps, and even given its limited size, it generated signals within the low-frequency regime, down to $2 \mathrm{~Hz}$, rather easily.
\end{abstract}

\section{INTRODUCTION}

The goal of a seismic vibrator is to exert a controlled time-varying force on the ground. In a basic vibrator, this force is provided by a driving engine that moves some (reaction) mass with respect to a base plate, which is in contact with the ground. To prevent the base plate from decoupling from the ground, a hold-down system might be present to add a static force on the base plate. A more detailed description of seismic vibrators can be found in Baeten and Ziolkowski (1990) and Meunier (2011). Seismic exploration vibrators are typically driven by a hydraulic engine. Although these engines can produce very large forces, the hydraulics put unwanted limitations on the vibrator. The intrinsic nonlinearity of the hydraulic system is one of the causes of the harmonics typically observed with vibrators (Sallas, 2010). Depending on the design, the hydraulic flow rate might (Sallas, 2010) or might not (Wei and Phillips, 2013) limit the output power of the vibrator at low frequencies. At the high end of the spectrum, the output is limited as well, due to the compressibility of the hydraulic fluid (Sallas, 2010). Furthermore, the storke, the maximal movement of the reaction mass possible, needed for the generation of low frequencies, and the volume and pressure within the hydraulic engine are directly related. Therefore, designing a vibrator with a larger stroke for more output at the low frequencies is not a trivial task.

Another possibility to drive a vibrator, which does not have the intrinsic limitations of a hydraulic system, is using a linear synchronous motor (LSM). An LSM is an electric motor able to generate linear forces and can be found in numerous applications. They are used in factories to move objects in a fast and precise way, but they can also be found in the propulsion system of some magnetic-levitation trains and roller coasters (Veltman et al., 2002). Use of an LSM to drive a seismic vibrator is proposed by Unger (2002) and Drijkoningen et al. (2006). The work of Drijkoningen et al. (2006) led to the development of the prototype LSM vibrator, which is presented in this paper. The main goal of this LSM vibrator is to show its feasibility as a seismic source. To keep the vibrator practical for research applications, it was kept relatively small, with a weight of about 1 ton and a driving force of about $7 \mathrm{kN}$. Still, it can well be used for shallow $(<1 \mathrm{~km})$ seismic exploration and is able to generate low frequencies down to $2 \mathrm{~Hz}$ at full force. Noorlandt et al. (2012) present some of the very first results obtained with this new vibrator.

Manuscript received by the Editor 24 June 2014; revised manuscript received 5 September 2014; published online 2 February 2015.

${ }^{1}$ Deltares, Department of Applied Geology and Geophysics, Utrecht, The Netherlands; Delft University of Technology, Department of Geoscience and Engineering, Delft, The Netherlands. E-mail: rik.noorlandt@deltares.nl.

${ }^{2}$ Delft University of Technology, Department of Geoscience and Engineering, Delft, The Netherlands. E-mail: g.g.drijkoningen@tudelft.nl.

${ }^{3}$ Seismic Mechatronics BV, Eindhoven, The Netherlands. E-mail: j.dams@ seismic-mechatronics.com; r.jenneskens@seismic-mechatronics.com.

(C) 2015 Society of Exploration Geophysicists. All rights reserved. 
In the present paper, we describe the design of the prototype LSM vibrator and the issues associated with building it. The operation of a single LSM and the synchronization of the six LSMs in the prototype vibrator is explained in some detail. The supporting structure of the prototype vibrator generates a few resonances, and we clarify their origin and a method to suppress them. Having explained the basic operation of the prototype LSM vibrator, some field data are presented. These data give insight to the harmonic behavior of the vibrator, the type of signals it can produce, and its ability to send out low-frequency signals. In the "Discussion" section, we describe the lessons learned and steps to be taken to build an LSM production vibrator.

\section{DESIGN AND PRINCIPLE OF A LINEAR-MOTOR VIBRATOR}

To show the feasibility of an LSM vibrator, a compact design was chosen that does not include a hold-down mass. In this way, the design is simpler and the number of possible elements that distort the signal is reduced. The disadvantage is that the vibrator force is limited to the weight of the reaction mass. Figure 1 shows a drawing of the vibrator and its components. Table 1 shows the basic properties of the vibrator. In addition to the base plate, reaction mass, and LSMs, it also contains an air spring and a few leaf springs. The air

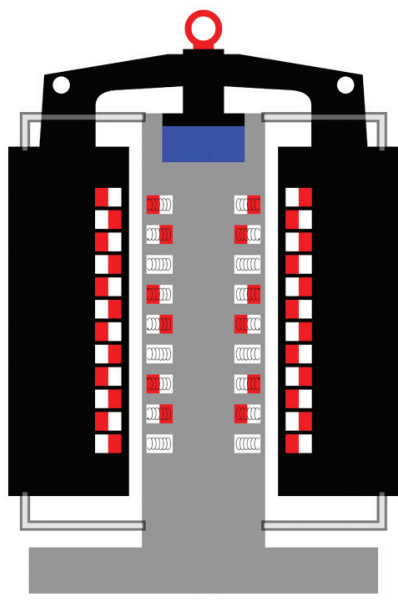

Reaction mass

Base plate / Stamp

Dermanent magnet

Dlectromagnet / Coils

Air spring

Leaf springs

Figure 1. A $2 \mathrm{D}$ sketch showing the different components of the LSM vibrator.

Table 1. Basic properties of the prototype LSM vibrator.

Reaction mass

$1027 \mathrm{~kg}$

Base-plate mass

$230 \mathrm{~kg}$

Base-plate area

$0.5 \mathrm{~m}^{2}$

Number of LSMs

6

Maximal driving force

$6.7 \mathrm{kN}$

Active stroke

$\pm 42 \mathrm{~mm}$

Lowest frequency at $100 \%$ drive level spring supports the reaction mass, thereby greatly reducing the total driving force needed. The leaf springs guide the reaction mass in the vertical direction, constraining the movement of the reaction mass to $1^{\circ}$ of freedom. The leaf springs provide a cost-effective way to guide the mass without adding any friction. Figure 2 shows the prototype vibrator deployed in the field.

\section{Mechanical modeling}

One of the most important design specifications of the vibrator is its frequency response. The amplitude response should be flat within a certain bandwidth, 2 to $200 \mathrm{~Hz}$ for our vibrator. To accomplish this, the dynamic behavior of the vibrator was predicted using finite-element modal analyses. The outcome of the finite-element simulation is used to create a continuous-time state-space model as described by Gawronski (2004), and this model is then used to analyze the frequency response for the (combination of) sensors and actuators. This procedure led to a few changes in the original design, removing some resonances within the frequency band of interest and making other resonances still in that band easier to control. As an example, Figure 3 shows the $25-\mathrm{Hz}$ rocking mode of the reaction mass that was clearly visible in one of the early designs. With the help of three, instead of one, vertical accelerometers on the reaction mass, this mode can be measured. This measurement is then used to balance the forces of the actuators, such that this mode is not excited, as will be shown later on.

Another very important aspect of a vibrator is its stroke, the amount of distance the reaction mass can move up and down. Given the weight of the reaction mass and the maximum force that the driving engine can produce, the stroke fixes the lowest frequency that the vibrator can produce at full force. If the reaction mass would only experience a driving force equal to $F=M A \sin (\omega t)$, where $M$ is the amount of mass, its displacement would follow $U=-\frac{A}{\omega^{2}} \sin (\omega t)$. In such a case, the displacement is related to the driving force by

$$
U=-\frac{F}{\omega^{2} M}
$$

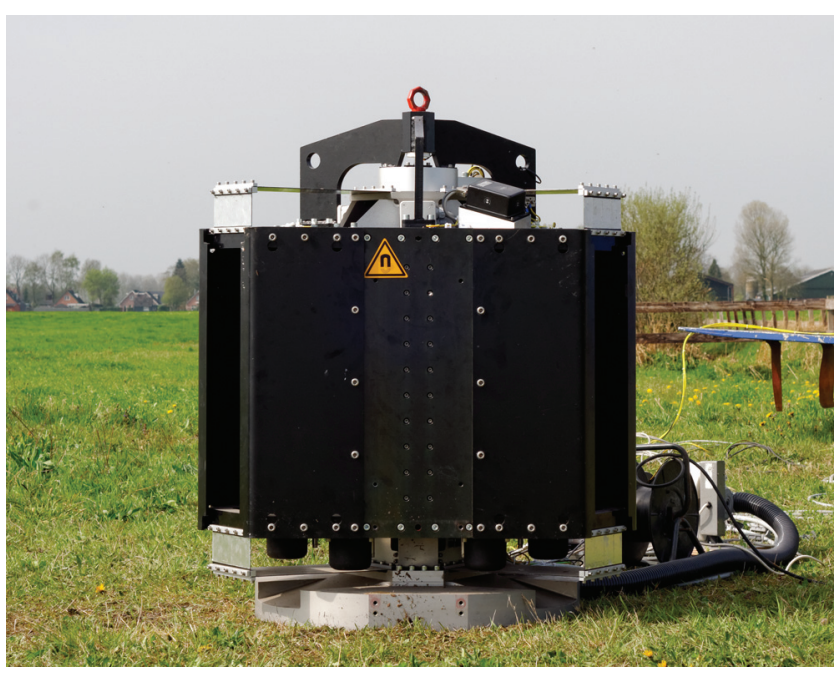

Figure 2. Prototype vibrator, based on LSMs, deployed in the field. 
So, for a fixed driving force and reaction mass, the displacement increases with decreasing frequency. To increase the maximum driving force at a fixed frequency, one has to increase the stroke, reaction mass, or both. For our prototype vibrator with its stroke, reaction mass, and maximum driving force, as given in Table 1 , the lowest frequency at full force is approximately $2 \mathrm{~Hz}$, equal to the design specification.

\section{Principle of a linear synchronous motor}

An LSM is an electric motor that can be seen as an unrolled permanent magnet synchronous motor. The LSM used in the prototype vibrator consists of a U-shaped permanent-magnet track and a coil track sliding in between, as shown in Figure 4. The intercoil and intermagnet distances are chosen such that the same force can be made for any position of the tracks by controlling the current distribution over the different coils. The need for the synchronization of the track positions and the current, together with the fact that the resulting force acts along a line, i.e., is linear, gives these types of motors their name. With this geometry, the motor can drive over any distance needed. Therefore, an LSM-driven vibrator will be able to equally generate low frequencies with a large stroke as high frequencies with a relative small stroke. This is in contrast to hydraulic engines in which fluid flow and dynamics limit and distort the output at low and high frequencies (Sallas, 2010), or single-coilmagnet designs in which the linearity is lost for larger amplitudes (Van Der Veen et al., 1999).

For a Lorentz-type motor, the force produced can be determined with the help of the Lorentz force law:

$$
\vec{F}=\int \vec{I} \times \vec{B} \mathrm{~d} l,
$$

where $\vec{F}$ is the force produced by the interaction of the current $\vec{I}$ flowing through the coil $\int \mathrm{d} l$ inside the magnetic field $\vec{B}$. In an ideal Lorentz motor, the magnetic field from the permanent magnets is perpendicular to the coil plane as seen in Figure 4. In equation 2, it is clear that the resulting force will, therefore, be in the plane of the coil. The distances between the straight parts of the coil $2 R_{C}$ in Figure 4 and between the magnets $R_{m}$ in Figure 4 are chosen to be the same. With such geometry, the force on both straight parts of the coil will be in the same direction. The alternating pattern of the permanent magnets causes the magnetic field strength to vary sinusoidally in the driving direction. It is relatively easy to show that with this setup, the total force on a single coil acts only in the driving direction $\hat{x}$ equal to

$$
\vec{F}(I, x)=G I \cos \left(\frac{\pi x}{R_{m}}\right) \sin \left(\pi \frac{R_{c}}{R_{m}}\right) \hat{x},
$$

where $x$ is the position of the center of the coil, $I$ is the current flowing through the coil, $G$ is a constant that depends on the magnetic field strength and the shape of the coil, and $R_{m}$ and $2 R_{c}$ are the distances between the magnets and straight part of the coil, as shown in Figure 4. Because the $R_{m}=2 R_{c}$, the sine term in equation 3 is equal to one.

To deal with the dependency on the coil position, multiple coils are used that are shifted by multiples of $\frac{2}{3} R_{m}$, as shown in Figure 4 . Together, these coils form three groups for which the total force is

$$
\begin{aligned}
\vec{F}\left(i_{1}, i_{2}, i_{3}, x\right) & =n G\left[i_{1} \cos \left(\frac{\pi x}{R_{m}}\right)+i_{2} \cos \left(\frac{\pi x}{R_{m}}+\frac{2 \pi}{3}\right)\right. \\
& \left.+i_{3} \cos \left(\frac{\pi x}{R_{m}}+\frac{4 \pi}{3}\right)\right] \hat{x},
\end{aligned}
$$

where $n$ is the number of coils per group and $i_{1}, i_{2}$, and $i_{3}$ are the currents applied to the different coil groups. To obtain a constant force, the currents applied to these groups need to be commuted with the same phase:

$$
\begin{gathered}
i_{1}=I \cos \left(\frac{\pi x}{R_{m}}\right), \\
i_{2}=I \cos \left(\frac{\pi x}{R_{m}}+\frac{2 \pi}{3}\right), \\
\text { and } \\
i_{3}=I \cos \left(\frac{\pi x}{R_{m}}+\frac{4 \pi}{3}\right),
\end{gathered}
$$

where $I$ is the magnitude of the current. Substituting the currents given by equation 5 in equation 4 gives

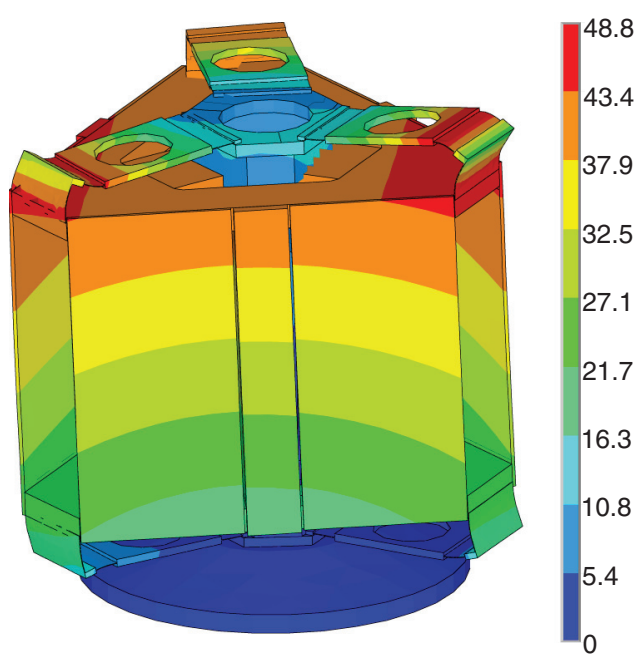

Figure 3. Finite-element prediction of the relative displacement of the vibrator at its $25-\mathrm{Hz}$ mode. Displacements are shown at their largest values, and the colors indicate the value of the mass normalized eigenvector (Gawronski, 2004).

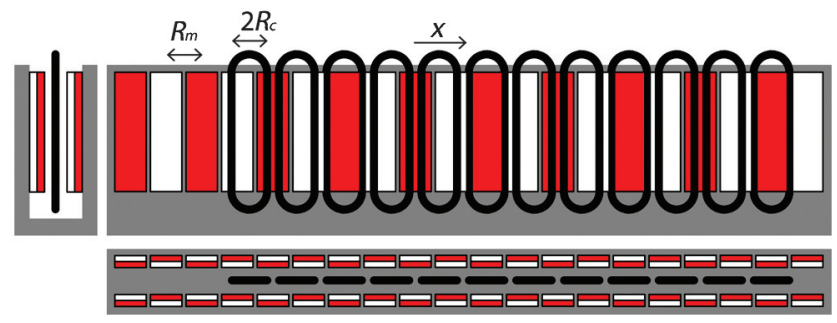

Figure 4. Sketch of the geometry inside the LSM motor. The two sides of the permanent magnets are colored in white and red, and the coils are black. 


$$
\vec{F}(I)=\frac{3}{2} n G I \hat{x} \quad=K I \hat{x},
$$

where $K$ is the so-called motor constant. The total force produced by the motor is thus linearly related to the applied current $I$. To make the force change over time, like a sweep, one simply divides the desired force-time signal by the motor constant and uses the output as the current input of the motor $I(t)=\frac{F(t)}{K}$.

Of course, there is a limit to the force that a single LSM can produce. Heat generation, proportional to coil resistance times the square of the current, was ignored in the above derivation. The generation of heat and the transport thereof sets the maximum current that the motor can endure before damage occurs and, therefore, sets the maximum force possible. During the design phase of our vibrator, the LSMs were carefully selected, balancing the maximum force, maximum stroke, efficiency, linearity, heat dissipation, and amplifier specifications. With the current motors and cooling design, the vibrator can be used continuously.

\section{Coil-magnet track synchronization}

For the LSM to work efficiently, the currents as expressed in equation 5 need to be in-phase with the cosine terms in equation 4 . Therefore, the displacement of the coils with respect to the magnetic field $x$ needs to be known, but the only displacement measurement available is between the reaction mass and base plate $u$. This measured displacement has an unknown offset $\Delta$ relative to $x$ that needs to be determined to be able to generate the currents of equation 5 .

To find $\Delta$, two currents, or forces if one multiplies with the motor constant $K$, are applied to the motor coils at the same time. The first set of currents applied to the coils is equal to the currents in equation 5, except that they are commuted with respect to the known $u$ instead of the unknown $x$. The second set of currents applied to the coils are used to distort the motors' behavior. They have a similar shape but a different amplitude and an extra phase offset $\alpha$ :

$$
\begin{gathered}
i_{1}=I_{A} \cos \left(\frac{\pi}{R_{m}} u\right)+I_{B} \cos \left(\frac{\pi}{R_{m}} u+\alpha\right), \\
i_{2}=I_{A} \cos \left(\frac{\pi}{R_{m}} u+\frac{2 \pi}{3}\right)+I_{B} \cos \left(\frac{\pi}{R_{m}} u+\frac{2 \pi}{3}+\alpha\right),
\end{gathered}
$$

and

$$
i_{3}=I_{A} \cos \left(\frac{\pi}{R_{m}} u+\frac{4 \pi}{3}\right)+I_{B} \cos \left(\frac{\pi}{R_{m}} u+\frac{4 \pi}{3}+\alpha\right) .
$$

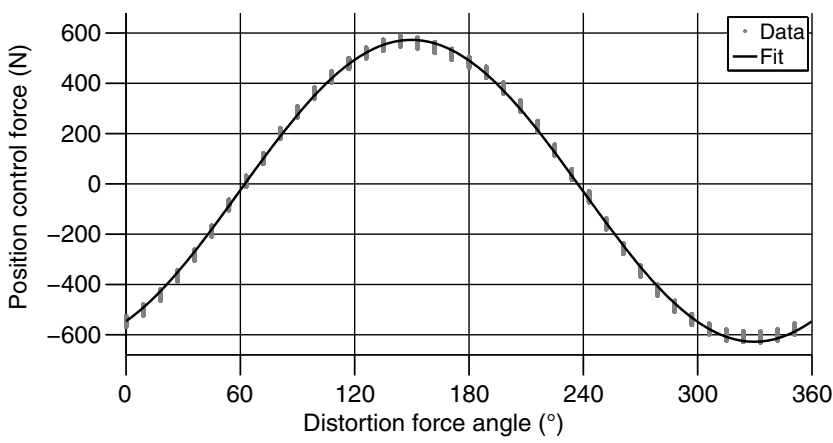

Figure 5. Position control force $K I_{A}$ versus distortion angle $\alpha$. The dots are measured data, and the line is the fit through these dots.
Substituting the currents given by equation 7 in equation 4 and making use of the fact that $u=x+\Delta$ gives

$$
\vec{F}\left(I_{A}, I_{B}, \alpha\right)=K I_{A} \cos \left(\frac{\pi \Delta}{R_{m}}\right) \hat{x}+K I_{B} \cos \left(\frac{\pi \Delta}{R_{m}}+\alpha\right) \hat{x} .
$$

The first term is the force of equation 6 , but the motor efficiency to convert electric current to force is reduced depending on the value of $\Delta$. Although the conversion efficiency of $I_{A}$ is fixed, the efficiency of converting the distortion current $I_{B}$ to force can be controlled with the phase offset $\alpha$. This can be used in a few ways to find $\Delta$. For our vibrator, we keep $I_{B}$ constant and vary $\alpha$ while a position controller is used to keep the reaction mass at the same position by changing $I_{A}$. Assuming that the motor force is constant at that fixed position, a change of $\alpha$ is completely compensated for by the controller current $I_{A}$. Therefore, by fitting multiple realizations of $I_{A}$ for different $\alpha$, as shown in Figure 5, it is possible to determine the unknown $\pi \Delta / R_{m}$ up to a multiple of $2 \pi$. With this, we can calculate $x$ and apply the currents given in equation 5, maximizing the output of the LSM.

\section{Multiple motor synchronization}

Depending on the desired driving force, multiple LSMs need to work in parallel. The motors cannot all be placed at the center of the vibrator and will, therefore, produce a moment with respect to the center of gravity. In our prototype vibrator, the motors are placed symmetrically, so that if they produce an equal force, all the moments cancel and only a net vertical force is left over. The motors and amplifiers are, however, not equally strong by default.

To find the correct gain for each amplifier-motor pair, use is made of three accelerometers located at the three edges of the reaction mass together with the rocking mode at $25 \mathrm{~Hz}$ as predicted by finite-element analysis (Figure 3 ). The rocking mode is clearly visible on the reaction-mass accelerometers, as shown in Figure 6, when the forces produced by the different LSMs are not balanced.

A grid search was performed varying the three motor gains between $90 \%$ and $100 \%$ to find gain values for which minimal rocking occurs and the forces from the linear motors are thus balanced. The results of the grid search are presented in Figure 7. It is clear that the minimum amount of rocking occurs if the gains of amplifier-motor pair 2 and 3 are reduced (Figure 7a). This means that for equal input, the force produced by the first amplifier-motor combination is less than that of the other two amplifier-motor combinations. Balancing the motor forces reduces the maximum average difference between the accelerometers' amplitude spectra by a factor of five and the different accelerometer signals are much more alike, as shown in Figure 8.

\section{Air spring support}

To avoid lifting the reaction mass to its neutral position with the LSMs and waste energy, a choice has been made to use an air spring between the reaction mass and base plate. The use of an air spring to bias the reaction mass to the center of its displacement range is also commonly found in hydraulic vibrators. If a hold-down mass is present, it is typically isolated from the base plate with an air spring as well. In both cases, the purpose of the air spring is to transfer the force at DC, without affecting the frequencies in the seismic band. 
The force produced by the air spring can be predicted from the ideal gas law. The air spring can be approximated by a closed cylinder with a volume $V$, for which the ideal gas law states that

$$
\frac{p V}{T}=k
$$

where $p$ is the pressure, $T$ is the absolute temperature inside the cylinder, and $k$ is a constant determined by the gas properties. The force the air spring exerts on the reaction mass is then given by

$$
F=k \frac{T}{h}
$$

where $h$ is the height of the cylinder. Equation 10 is only valid as long as the cylinder can be compressed without changing its diameter or contact area with the reaction mass.

A Taylor expansion around the neutral cylinder height $h_{0}$ gives

$$
\begin{aligned}
F= & \frac{k T}{h_{0}}-\frac{k T}{h_{0}^{2}}\left(h-h_{0}\right)+\frac{k T}{h_{0}^{3}}\left(h-h_{0}\right)^{2} \\
& +\mathcal{O}\left(\left(h-h_{0}\right)^{3}\right) \quad\left|h-h_{0}\right|<1,
\end{aligned}
$$

where use was made of the fact that the reaction-mass displacement is related to the cylinder height, through $\left(h-h_{0}\right)$. From equations 10 and 11, it is clear that the air spring behaves nonlinearly as a function of $h$ and is temperature dependent.

The air spring for the prototype vibrator was selected such that the first Taylor term compensates for the gravity force, the resonance frequency of the spring is below $2 \mathrm{~Hz}$, it has low damping, and it meets certain safety regulations. Although the resonance itself is outside the designed bandwidth, at approximately $1.5 \mathrm{~Hz}$, it still has a significant influence to the response up to some $6 \mathrm{~Hz}$, as can been seen in Figure 8.

\section{Suppressing support spring resonance and temperature effects}

Because the spring resonance frequency is low, the driving force at this frequency must be limited to prevent exceeding the available stroke. To suppress the resonance behavior, two different control methods were implemented.

First, a feed-forward control was tried, changing the driving force in advance to anticipate the spring response. Although this method is theoretically more stable than the feedback method described below, it was not successful in suppressing the resonance. This mainly had to do with the hysteresis of the air spring, most probably caused by the deformation of the rubber air container, making a prediction of the exact spring force very difficult.

Therefore, use is made of a position feedback control. The position controller changes the driving force in real time such that the reaction-mass displacement follows the prescribed position curve as closely as possible. The position curve is calculated beforehand based on the pilot and desired dynamics. To prevent base-plate resonance signals from getting into the control loop, the position control is only active for low frequencies. With this setup, the LSMs try to remove any low-frequency influence the springs have on the system. A nice side effect of using a position feedback is that any temperature effect of the air spring, as described previously, is also suppressed by this controller.

As an example, the position feedback controller was used to make the reaction mass move as if gravity and the spring forces were absent, and the only force acting was the force from the LSMs. In that case, the position controller suppresses the behavior of the springs. The reaction-mass displacement belonging to the driving force only can be found by dividing the sweep force by the reaction mass and integrating it twice. We simply used the trapezoidal method on the heavily oversampled time signal for this. Figure 9 shows reaction-mass displacement without and with the position controller active. It is clear that, for this example, the displacement amplitude caused by the support system is larger than that of the LSMs. When the position controller is active, as shown in Figure 9b,
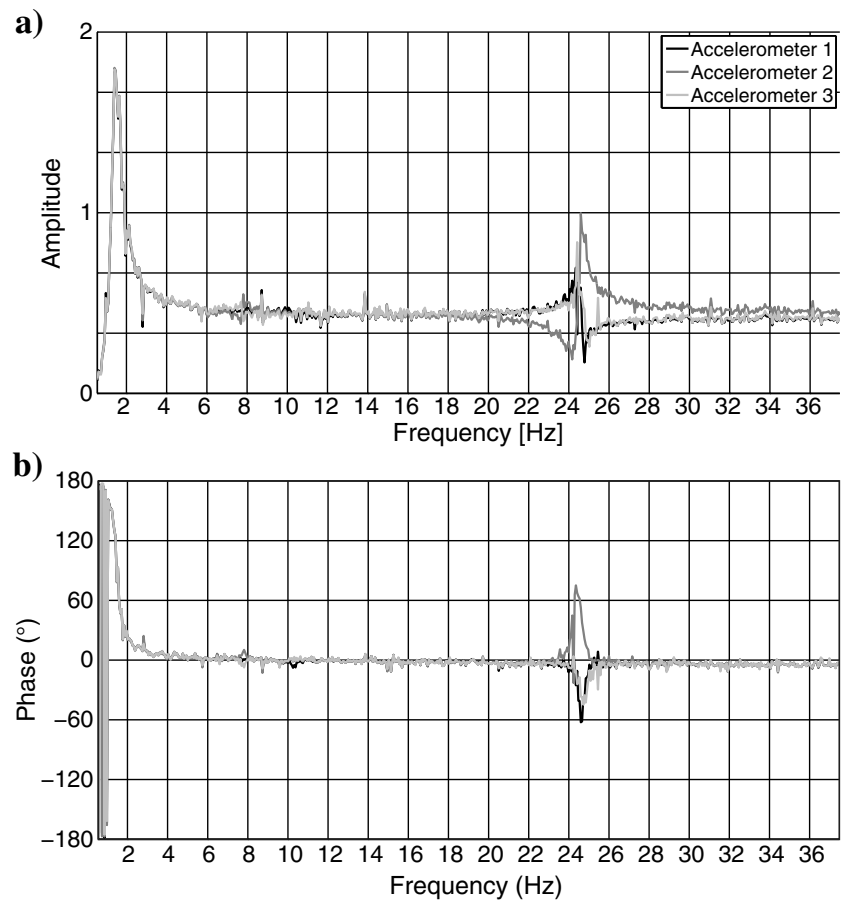

Figure 6. Example of the (a) amplitude and (b) phase response measured by the three reaction-mass accelerometers, when the three motor forces are unbalanced. a)

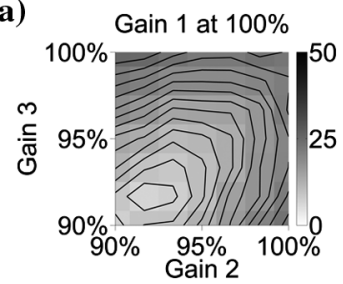

b)

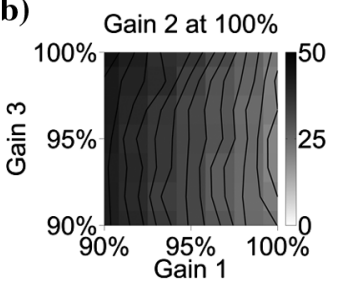

c)

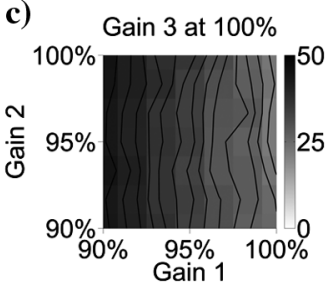

Figure 7. Influence of the amplifier-motor gain distribution on the difference among the reaction-mass accelerometers' responses. Colors indicate the sum of the absolute difference among amplitude spectra of each pair of reaction-mass-accelerometer signals between 20 and $30 \mathrm{~Hz}$. Each panel shows the result of scanning two gains, and the third one was kept at $100 \%$. 
the LSMs suppress the spring behavior very well. The reactionmass displacement follows the prescribed position curve closely, and the average absolute difference between the two curves is reduced by a factor of approximately 160 .
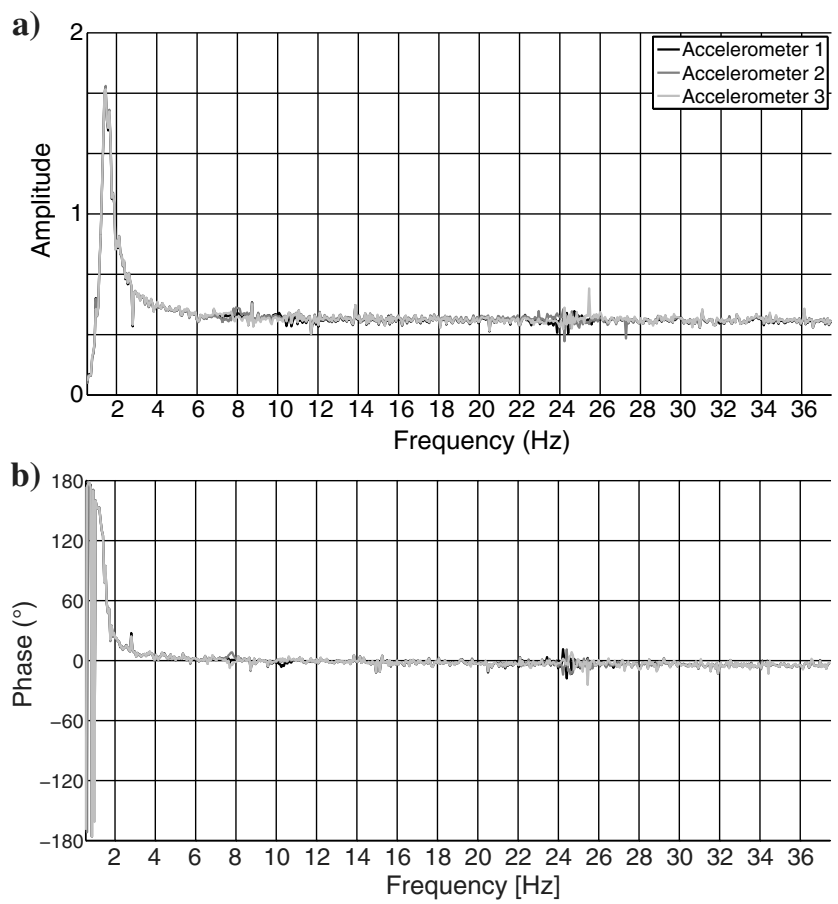

Figure 8. (a) Amplitude and (b) phase response measured by the three reaction-mass accelerometers, when the three motor forces are balanced. Note that comparing these results with that of Figure 6, the air spring resonance at approximately $1.5 \mathrm{~Hz}$ is not affected by motor balancing, whereas the rocking mode at approximately $25 \mathrm{~Hz}$ is.

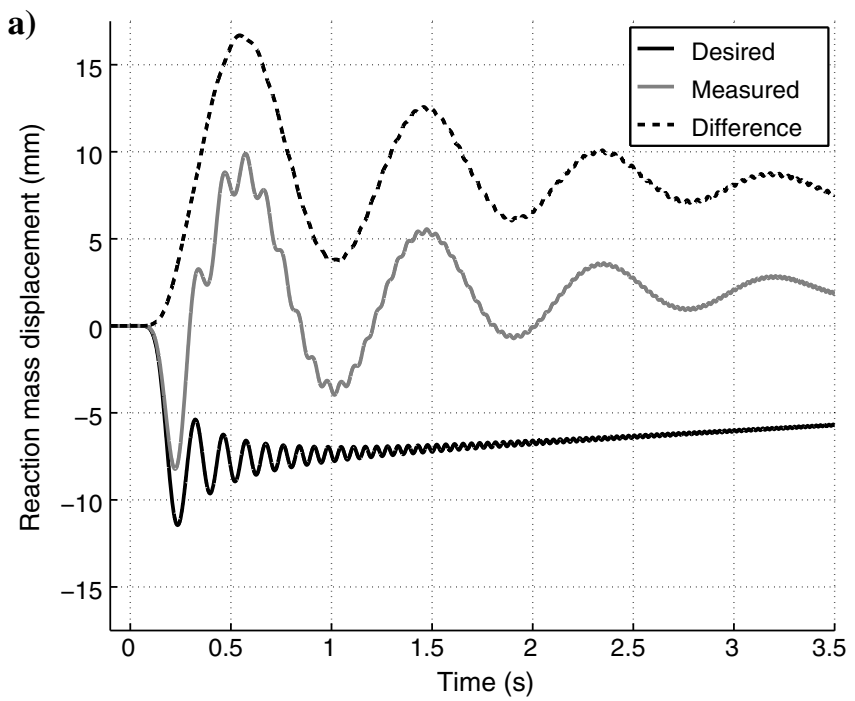

\section{FIELD CASE}

To show the seismic performance of the vibrator, we carried out some field tests at a seismic-monitoring site in the northeast of the Netherlands. At this site, $4 \mathrm{C}$ sensors, each equipped with a $3 \mathrm{C}$ geophone and one hydrophone, are buried at a fixed level with respect to the geoid at approximately $50 \mathrm{~m}$ below the surface. In this paper, only the data from the hydrophones and vertical component of the geophones are presented. During the field tests, we also temporarily installed a few surface geophones. All the recorded data were correlated with the pilot signal. This was done to keep all distortion caused by the vibrator visible in the seismic records. It also prevented any noise from the accelerometers to propagate into the records. The accelerometer's measurements, however, are used to show the harmonic and low-frequency behavior of the vibrator.

\section{Regular linear sweep}

The most common signal to drive a seismic surface vibrator is the linear upsweep, in which the frequency of the driving sinusoid is increased linearly with time. The signal is tapered or faded at both ends to avoid step behavior. Figure 10 shows the seismic record obtained with a 10-s linear sweep from 5 to $200 \mathrm{~Hz}$, after being correlated with the pilot channel. The buried geophones and hydrophones show the direct arrival and a few reflections. Even though the geophones are at an approximately 50-m depth, they still pick up Rayleigh-wave energy. The hydrophones have a different sensitivity and, therefore, their record is less noisy and does not show the Rayleigh-wave arrival that strongly. Both records have some ringing, which indicated that the correlation with the pilot signal is not fully compressing the source wavelet in the seismic data.

\section{Harmonics}

Signal distortion is an important issue with seismic vibrators. Especially the generation of harmonics is a common and difficult problem (Seriff and Kim, 1970). For a linear upsweep, the energy

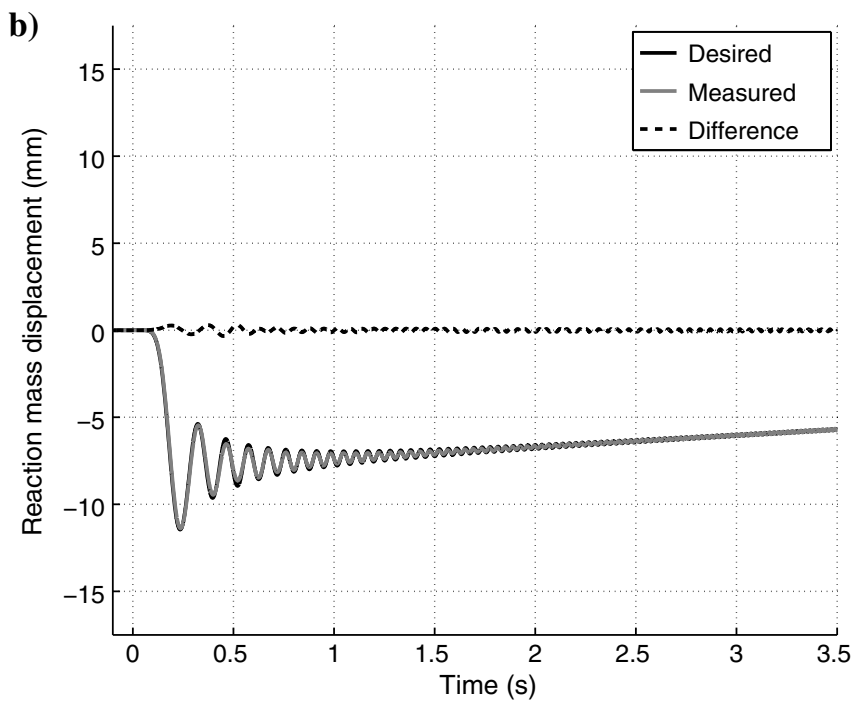

Figure 9. The first $3.5 \mathrm{~s}$ reaction-mass displacement for a 12-s linear sweep from 2 to $160 \mathrm{~Hz}$. (a) Without position feedback control and (b) with position feedback control. 
of the harmonics is mapped to earlier arrival times, possibly masking earlier events. To investigate the distortion and harmonics of the prototype vibrator, the acceleration of the reaction mass (average of the three sensors), the acceleration of the base plate, and the weighted-sum ground force are used. The weighted-sum ground force (Castanet and Lavergne, 1965; Sallas, 1984) estimates the force that the vibrator exerts on the ground by summing the acceleration of the reaction mass and base plate, weighted with their masses. In a rigid $1 \mathrm{D}$ approximation, the reaction mass only experiences the force from the driving engine, whereas the base plate experiences the force from the driving engine (with opposite sign) as well as the force from the ground. By summing, one thus removes the influence of the engine and is left with the force on the ground only.

It is expected that LSMs produce less harmonics compared with hydraulic engines because they act more linearly. The mechanics and ground coupling, however, also generate harmonics. Therefore, the influence of the driving engine alone on the total signal distortion cannot be determined.

Figure 11 shows the time-frequency analysis for the pilot signal, the measured accelerations, and the weighted-sum ground force. Next to the designed signal, these plots also show energy at other times and frequencies. The harmonics of the weighted-sum ground force, Figure $11 \mathrm{~b}$, are on the order of $20 \mathrm{~dB}$ lower than the fundamental signal at all frequencies, including the lower. If we look at the signals that make up the weighted-sum ground force, the reaction-mass acceleration, shown in Figure 11c, and base-plate acceleration, shown in Figure 11d, we see that most of the weighted-sum ground-force harmonics originate from the base-plate acceleration signal. The reaction-mass harmonics are at approximately $-30 \mathrm{~dB}$, whereas the base-plate harmonics are already visible at $-20 \mathrm{~dB}$. This shows that the LSMs have a limited contribution to the harmonics found in the weighted-sum ground force. Most of the harmonics are coming from the base plate, which might be caused by the ground coupling. It is striking that the recorded pilot signal, shown in Figure 11a, shows harmonics as well, although these are not in the designed pilot signal. These harmonics are probably caused by the electromagnetic (EM) interference between the amplifiers and our recording equipment. If this is the case, part of the harmonic energy observed with the accelerometers actually does not originate from the movement of the masses.

\section{Other driving signals}

The first step in processing the seismic records obtained with a vibrator is removing the phase of the source signal from the seismic response, thus compressing the record as if the vibrator had sent out
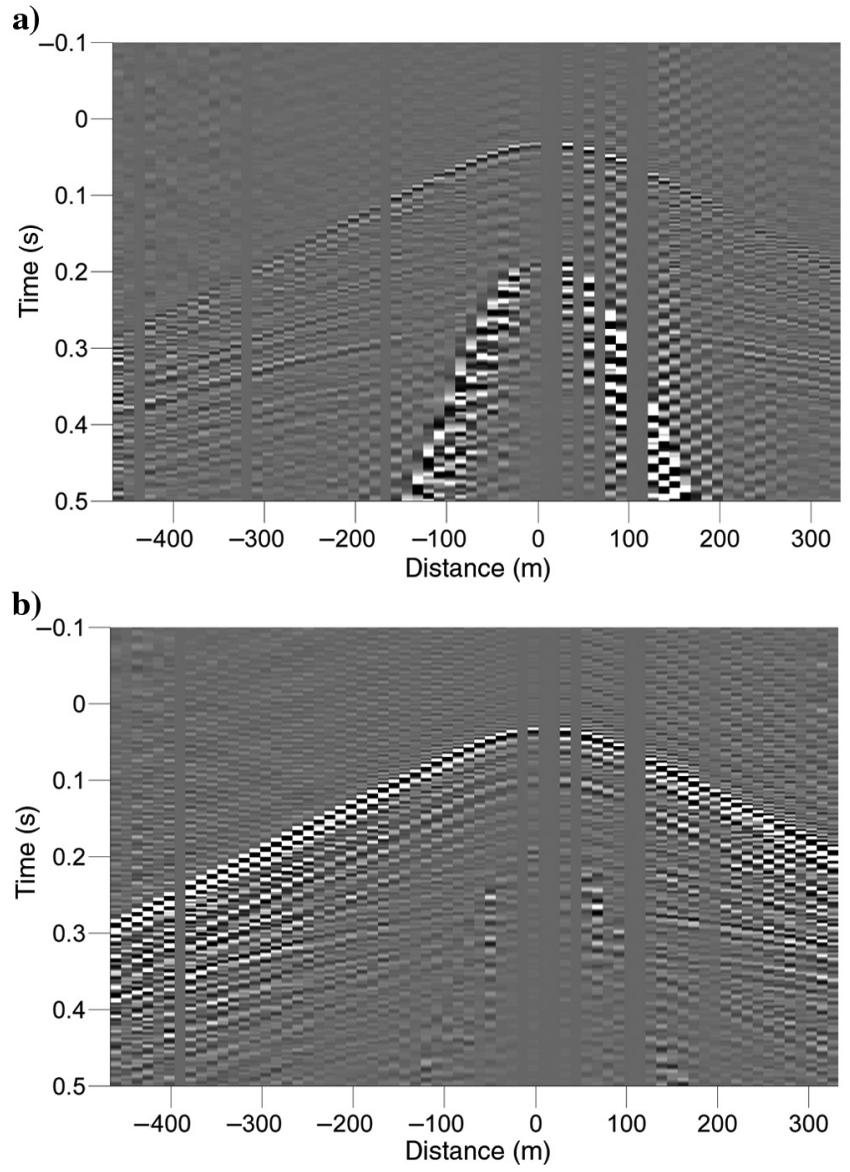

Figure 10. Seismic records obtained at 50-m depth with a 10-s linear sweep from 5 to $200 \mathrm{~Hz}$. Vertical geophone data are shown in panel (a), and hydrophone data are shown in panel (b). (Dead traces are blanked.)
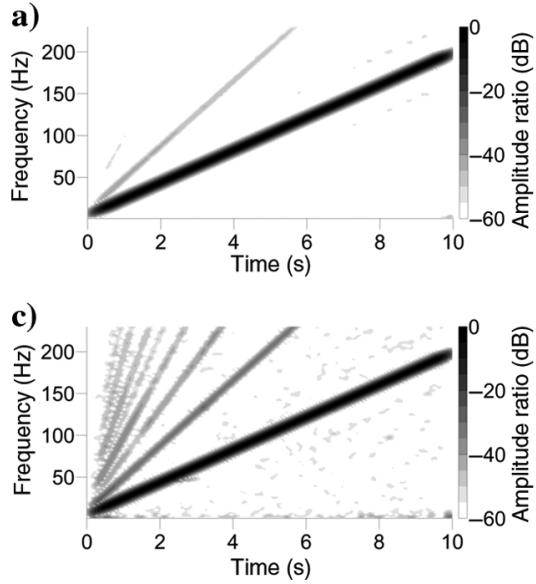

b)

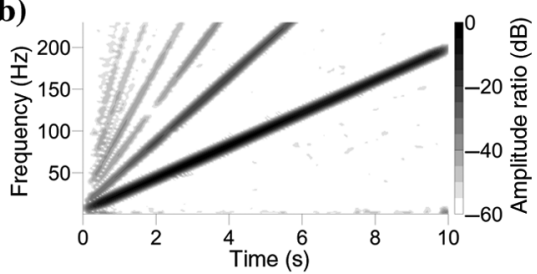

d)

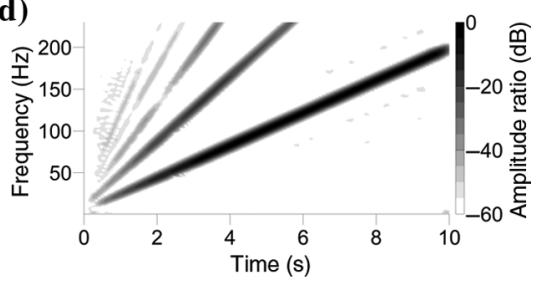

Figure 11. (a) Time-frequency plot of the pilot signal, (b) weighted-sum ground force, (c) reaction-mass acceleration (after time averaging the three sensors), and (d) base-plate acceleration. 
a zero-phase wavelet. The phase of the source wavelet, therefore, can be changed at will, without affecting the seismic record. This opens up the possibility to design signals with specific properties. One of these properties might be designing multiple signals that are orthogonal to each other, i.e., have low crosscorrelation. With such signals, multiple seismic vibrators could work simultaneously reducing the acquisition time tremendously. Pseudorandom signals (for an overview, see Dean, 2014) can be designed to have such properties. One of the reasons why pseudorandom signals are not used that frequently is the difficulty to transmit them by (hydraulic) vibrators (Dean, 2014).

To show that the LSM vibrator has no problem with producing this kind of signal, we randomized the phase of a linear upsweep and compared the seismic records obtained with the original sweep and randomized one. The signals are shown in Figure 12. Because the amplitude spectra are the same, both signals have the same autocorrelation. However, the time and time-frequency behaviors are quite different. In the time domain, the envelopes of both signals are quite different, and the randomized sweep shows peaks that are approximately three times larger than the amplitude of the linear sweep. Although there is a simple one-to-one mapping from time to frequency for the linear sweep, there is no such relation for the randomized signal as is visible in the time-frequency plot. Figure 13 shows the obtained buried-geophone records. Because we maximized the force to approximately $6 \mathrm{kN}$, while keeping the amplitude spectra the same, the time-domain peaks of the randomized signal cause the seismic signal-to-noise ratio to be lower than the records as shown in Figure 10. The reflections are, however, still visible in
Figure $13 \mathrm{a}$ and $13 \mathrm{~b}$. The difference between the records is minimal and was most likely caused by the difference in noise and the ability to fully compress the source wavelet by correlation with the pilot signal.

To show the ability of the vibrator to send low frequencies, a randomized phase signal with a very steep slope in the amplitude spectrum was designed. This slope makes it possible to have a strong signal at $2 \mathrm{~Hz}$ without having the air-spring resonance consume all of the available stroke. A maximal force of approximately $6 \mathrm{kN}$ was used again. The spectra measured at the vibrator, shown in Figure 14, show that the reaction mass (Figure 14c) follows the designed signal (Figure 14a) closely. Because we do not apply feedback on the weighted-sum ground force, the base-plate acceleration (Figure 14d) changes the weighted-sum ground force spectra (Figure 14b) at the higher frequencies. Figure 15 shows the mean ambient noise spectra recorded with the surface geophones and the response curves belonging to these geophones. From this, it is clear that a larger amount of energy of the vibrator is needed for low frequencies to overcome the ambient noise level and lower geophone response. Figure 16 shows the seismic record acquired with the low-frequency signal of Figure 14. Note that no correction was applied for the geophone response. Different low-pass filters were applied to the data. Figure 16a shows the record without applying a low-pass filter. In Figure 16b-16d, low-pass filters with a cutoff at 4, 6, and $8 \mathrm{~Hz}$ were applied. Most ambient noise enters the record from the right as is clearly visible below the surface wave in the 4$\mathrm{Hz}$ version of the record, possibly originating from the road and farms on that side of the line. The surface waves from the LSM a)

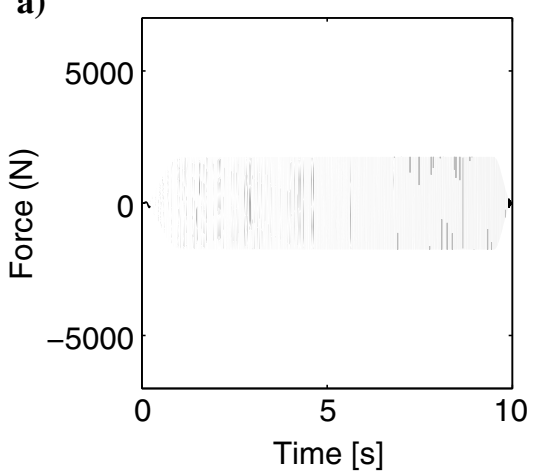

d)

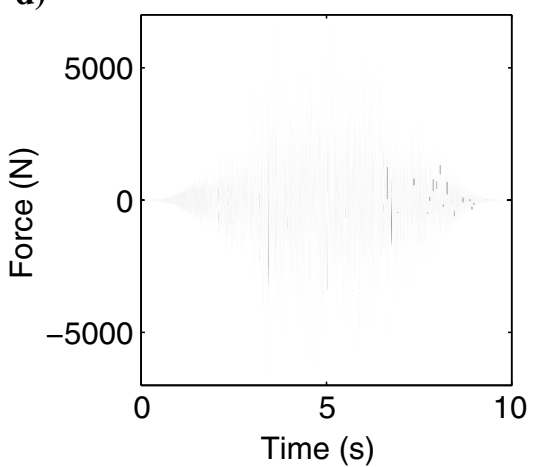

b)

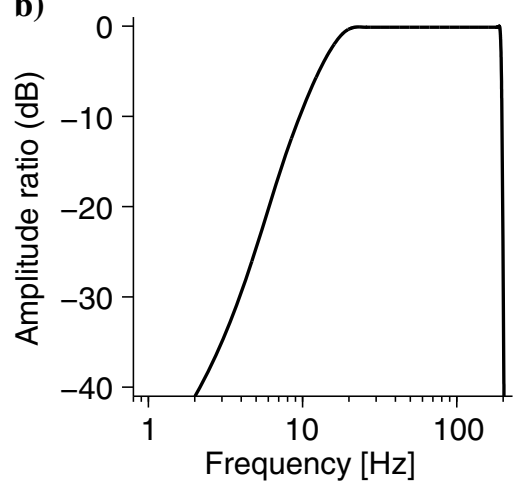

e)

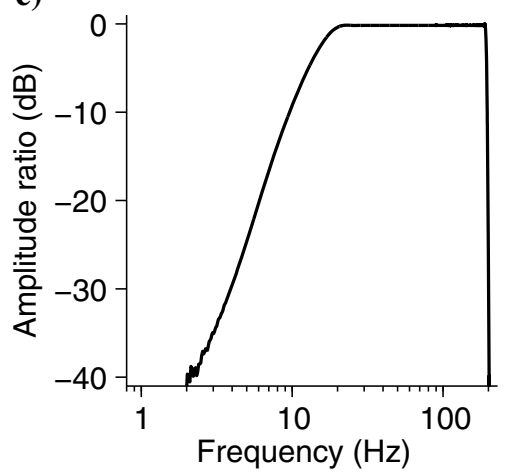

c)

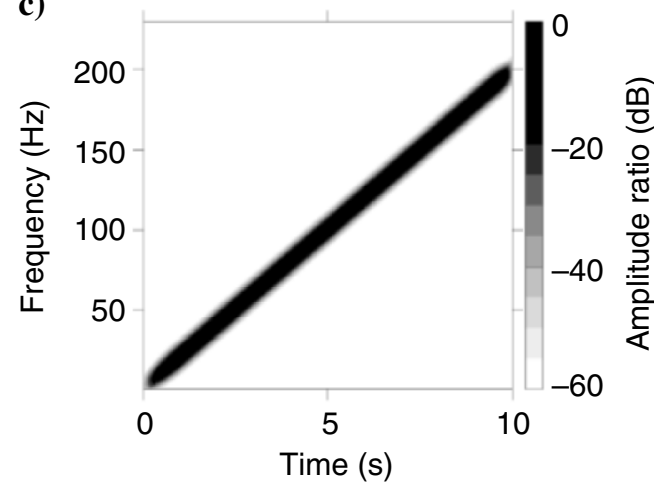

f)

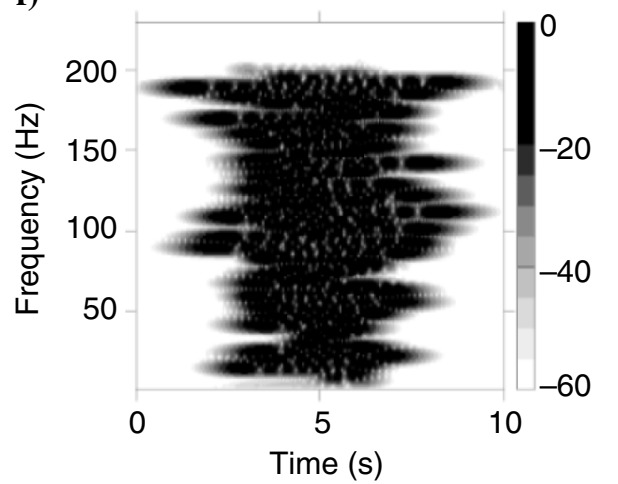

Figure 12. Regular linear upsweep (top) and a power-spectrum equivalent pseudorandom signal (bottom). (a and d) Time-domain signal, (b and e) amplitude spectra, and (c and f) time-frequency plot. Seismic records belonging to these signals are shown in Figure 13. 
vibrator are visible in all versions of the record, but with increasing filter bandwidth, they become more dominant with respect to the ambient noise, as is expected from Figure 15.

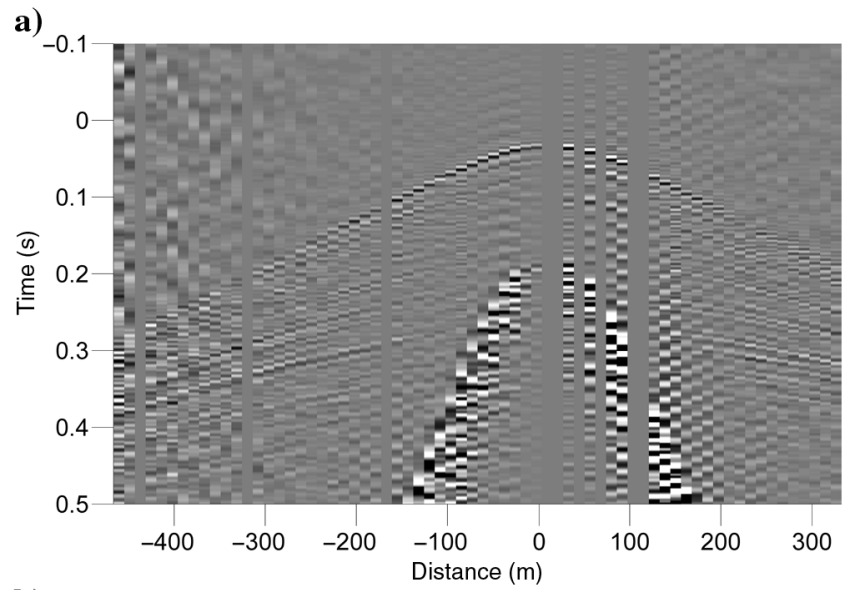

b)

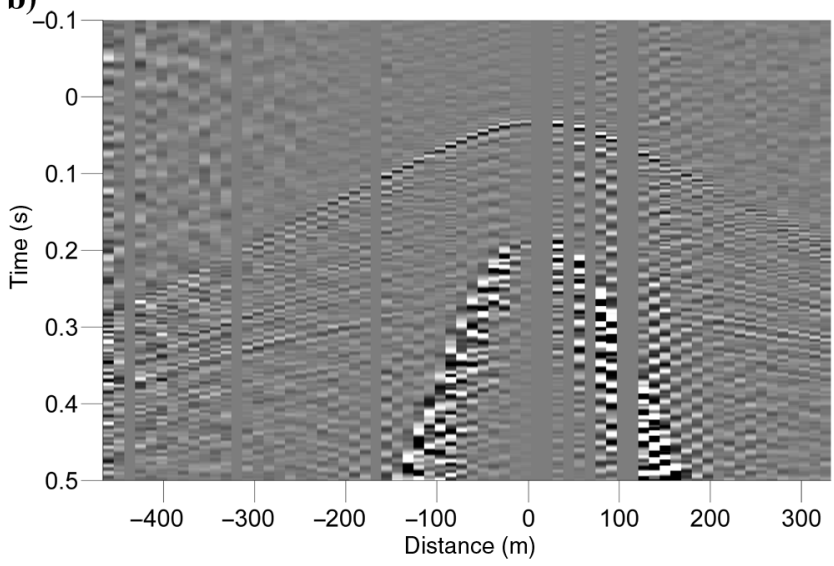

Figure 13. Seismic records obtained with the vertical geophones at a 50-m depth. (a) Response of the regular sweep and (b) the response of the pseudorandom signal. (Dead traces are blanked.)

\section{DISCUSSION}

As with every prototype, one gains much insight going through the process of actually building and testing it. Many choices were made and reconsidered during the design and building phase of the vibrator presented. With the knowledge that we now have, we would make two choices differently. First, we would use an air spring with a larger volume, increasing $h 0$ in equation 11 . By doing so, the vibrator would need to get a different safety certification, but the mechanical resonance frequency would be lower and the temperature sensitivity would be smaller. This would allow for easier generation of low frequencies, with less need of a position controller. We would also opt for a different type of amplifier and change the wiring of the system. In the current prototype, six pulse-modulated amplifiers are used to power the LSMs. These amplifiers generate the currents needed by switching on and off a high-voltage source at $20 \mathrm{kHz}$. This binary high-voltage sequence is then smoothed by passing it through a low-pass filter before it goes to the LSM. These types of amplifiers are very efficient, but the drawback is that they generate EM noise. This EM noise, together with how the vibrator is wired, causes signal distortion that is picked up by the accelerometers. With better amplifiers, the harmonics visible in Figure 11 can be reduced significantly. This is supported by data from a smaller LSM vibrator recently built (see Drijkoningen and Noorlandt, 2014). That vibrator does not have an air spring because the movement is perpendicular to the gravity force. It only contains two LSMs to drive the reaction mass and uses linear amplifiers to drive them. The harmonics observed with this vibrator, as shown in Figure 17, are extremely small: The recorded pilot signal is free of harmonics, and the harmonics visible in the weighted-sum ground force signal are $30 \mathrm{~dB}$ below the fundamental signal. These harmonics completely come from the base plate because the reaction mass is free of harmonics up to $-80 \mathrm{~dB}$. The low-frequency distortion originates from the support springs and has an amplitude of $-45 \mathrm{~dB}$. This shows that LSM-driven vibrators can indeed reproduce a designed wavelet, including the low frequencies, faithfully.

The main purpose of our prototype LSM vibrator is to show the feasibility of an LSM-driven vibrator. The current vibrator can be a)

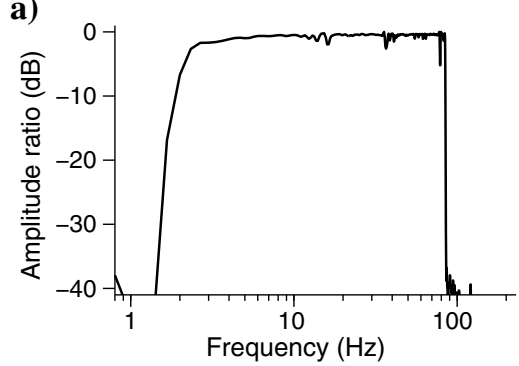

c)

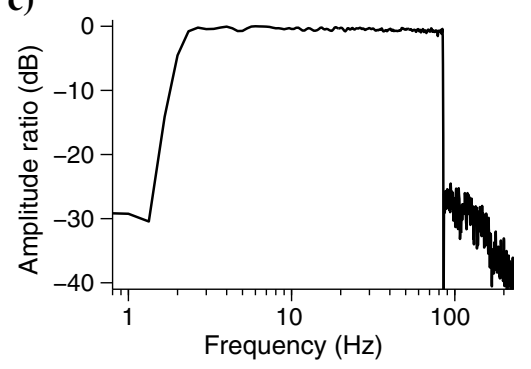

b)

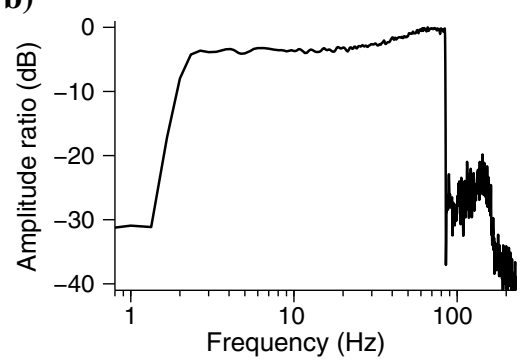

d)

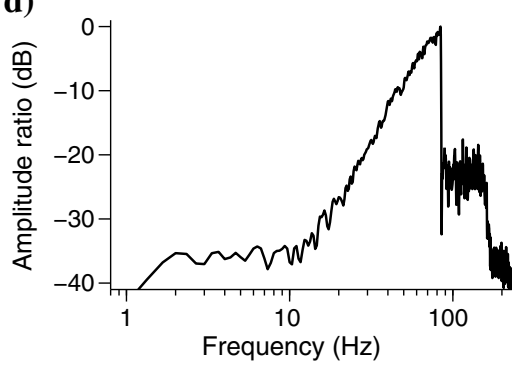

Figure 14. Pseudorandom signal with a very sharp low cut in the frequency domain. (a) Pilot signal, (b) weighted-sum ground force, (c) reaction-mass acceleration (after time averaging the three sensors), and (d) base-plate acceleration. The seismic record belonging to this signal is shown in Figure 16. 
used for shallow monitoring studies (Arts et al., 2013). For deeper targets (deeper than $1 \mathrm{~km}$ or so) and larger offsets, the current force is not large enough. To upscale, more or stronger LSMs would be

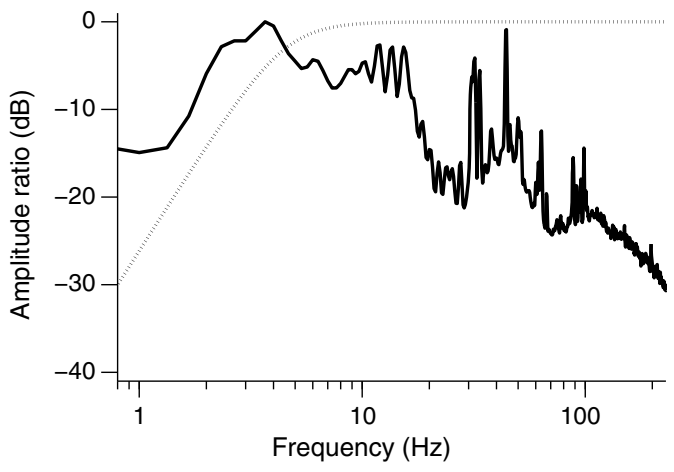

Figure 15. Spectra of the background noise (solid line) and the 4.5-Hz geophone response (dashed line). needed. They are readily available, but the circular design might not be well suited to position a large number of LSMs. The radius of the vibrator has to increase, which makes it more sensitive for rocking due to the longer leaf springs and the increased moments of the engines. A rectangular design could be more suitable in that case. Depending on the frequency range desired, the stroke and reaction mass should be changed as well. If the source should be used for exploration depths of a few kilometers, with a lot of source positions, the portability should be enhanced as well. The prototype vibrator can be lifted as soon the base plate is manually locked to the reaction mass. This locking should be made automatically, or one should change the base plate such that it can be lifted directly to reduce time needed to reposition the source. Of course, having a dedicated vibrator truck, as typical exploration vibrators have, would allow operation in the field to speed up even more.

\section{CONCLUSION}

LSMs can be used to drive a seismic vibrator. We successfully designed and built a prototype LSM vibrator, with which we
Figure 16. Seismic waves as recorded by the surface geophones using the signal shown in Figure 14. (a) Unfiltered, (b) low pass up to $4 \mathrm{~Hz}$, (c) low pass up to $6 \mathrm{~Hz}$, and (d) low pass up to $8 \mathrm{~Hz}$. Surface waves coming from the vibrator are marked with a black line above and below their arrival. (Dead traces are blanked.)

Figure 17. Time-frequency plot of the (a) pilot signal, (b) weighted-sum ground force, (c) reaction-mass acceleration, and (d) base-plate acceleration obtained with a small LSM vibrator (Drijkoningen and Noorlandt, 2014). Note that the color scale extends to $-80 \mathrm{~dB}$ in contrast to the $-60 \mathrm{~dB}$ scale used in Figure 11.
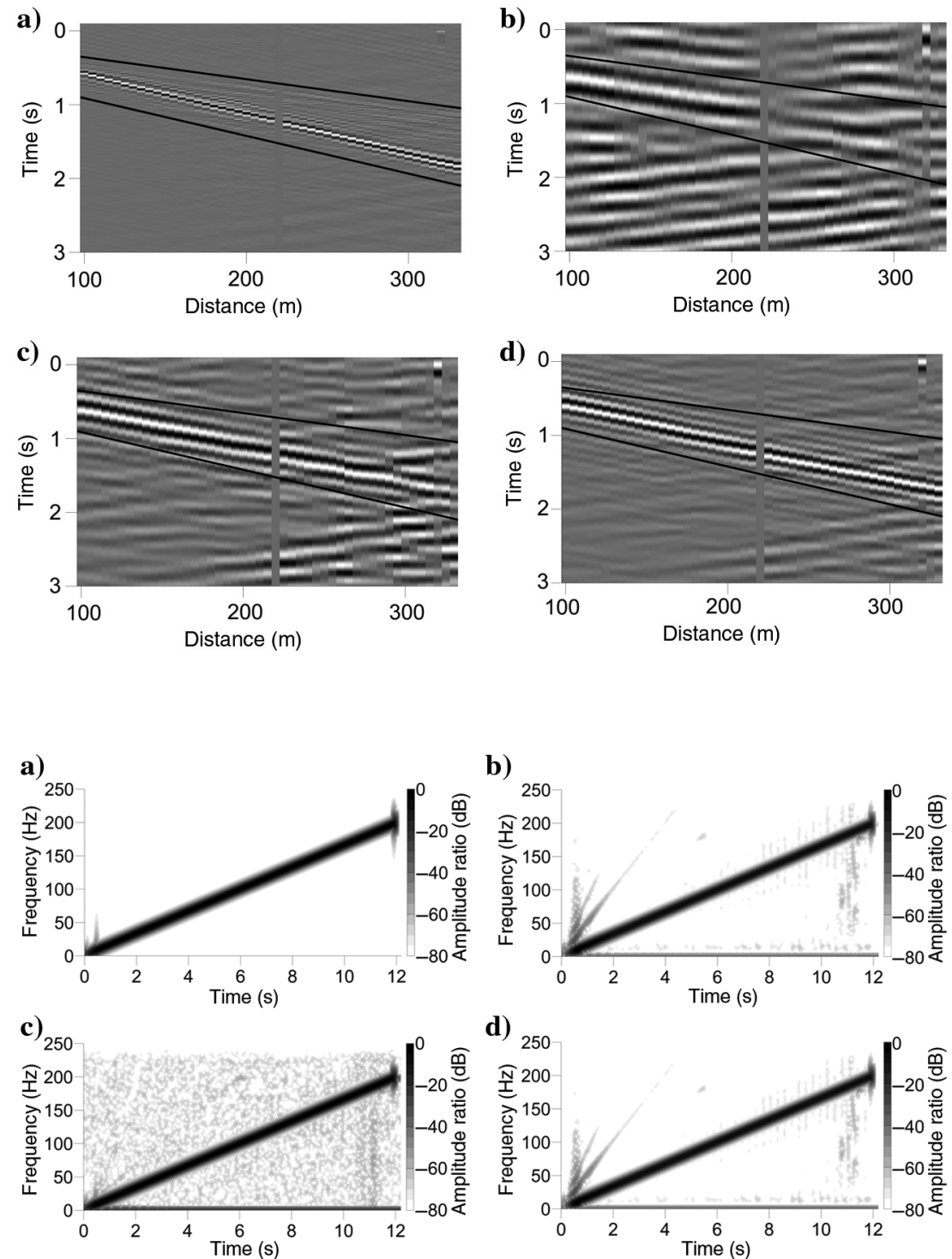
acquired good seismic data using a wide range of signals. The prototype LSM vibrator easily generates low-frequency signals as well as pseudorandom signals, which makes it a good candidate for simultaneous sweeping. The difference between an LSM and a hydraulic engine was explained, and the steps to be taken to build a production version were discussed.

\section{ACKNOWLEDGMENTS}

We thank LOFAR for allowing us to use the permanent seismic array and presenting the data. This work was sponsored by the Research School of Integrated Earth Sciences. It was also supported by the Nederlandsche Aardolie Maatschappij and Deltares.

\section{REFERENCES}

Arts, R., X. Zhang, A. Verdel, D. Santonico, J. Meekes, R. Noorlandt, B Paap, and V. Vandeweijer, 2013, Experiences with a permanently installed seismic monitoring array at the $\mathrm{CO}_{2}$ storage site at Ketzin (Germany), a status overview: Energy Procedia, 37, 4015-4023, doi: 10.1016/j.egypro .2013.06.301.

Baeten, G., and A. Ziolkowski, 1990, The vibroseis source: Elsevier.

Castanet, A., and M. Lavergne, 1965, Vibrator controlling system: U.S. Patent $3,208,550$

Dean, T., 2014, The use of pseudorandom sweeps for vibroseis surveys: Geophysical Prospecting, 62, 50-74, doi: 10.1111/1365-2478.12074.

Drijkoningen, G., and R. Noorlandt, 2014, Pseudo-random signals and EMtype seismic vibrators: Presented at Nederlands Aardwetenschappelijk Congres - NAC12.
Drijkoningen, G., A. Veltman, W. Hendrix, K. Faber, J. Brouwer, and G. Hemstede, 2006, A linear motor as seismic horizontal vibrator: 68th Annual International Conference and Exhibition, EAGE, Extended $\mathrm{Ab}$ stracts, P072.

Gawronski, W. K., 2004, Advanced structural dynamics and active control of structures: Springer.

Meunier, J., 2011, Seismic acquisition from yesterday to tomorrow: SEG, Distinguished Instructor Series.

Noorlandt, R., G. Drijkoningen, and R. Schneider, 2012, A seismic vertica vibrator driven by linear motors: 74th Annual International Conference and Exhibition, EAGE, Extended Abstracts, P061.

Sallas, J., 1984, Seismic vibrator control and the downgoing P-wave: Geophysics, 49, 732-740, doi: 10.1190/1.1441701.

Sallas, J., 2010, How do hydraulic vibrators work? A look inside the black box: Geophysical Prospecting, 58, 3-18, doi: 10.1111/j.1365-2478.2009 .00837.x.

Seriff, A., and W. Kim, 1970, The effect of harmonic distortion in the use of vibratory surface sources: Geophysics, 35, 234-246, doi: 10.1190/1 .1440087.

Unger, R., 2002, A seismic transmission system for continuous monitoring of the lithosphere: A proposition: Ph.D. thesis, Delft University of Technology.

Van Der Veen, M., J. Brouwer, and K. Helbig, 1999, Weighted sum method for calculating ground force: An evaluation by using a portable vibrator system: Geophysical Prospecting, 47, 251-267, doi: 10.1046/j.1365-2478 1999.00133.x.

Veltman, A., P. van der Hulst, M. Jonker, and J. van Gurp, 2002, Control of a 2.4MW linear synchronous motor for launching roller-coasters: Presented at 17 th International Conference on Magnetically Levitated Systems and Linear Drives, PP07301.

Wei, Z., and T. F. Phillips, 2013, On the generation of low frequencies with modern seismic vibrators: Geophysics, 78, no. 2, WA91-WA97, doi: 10 .1190/geo2012-0342.1. 